\title{
Comparison of effectiveness and safety of direct oral anticoagulants versus vitamin-k antagonists in elderly patients with atrial fibrillation: a systematic review and cost-effectiveness analysis protocol
}

\author{
Na Wang ${ }^{1,2}$, Nan-Nan Shen ${ }^{3}$, Yue Wu ${ }^{4}$, Chi Zhang ${ }^{2}$, Mang-Mang Pan ${ }^{2}$, Yan Qian ${ }^{1}$, Zhi-Chun Gu ${ }^{2}$ \\ ${ }^{1}$ Department of Pharmacy, The Second Affiliated Hospital of Chongqing Medical University, Chongqing 400010, China; ${ }^{2}$ Department of Pharmacy, \\ Renji Hospital, School of Medicine, Shanghai Jiao Tong University, Shanghai 200127, China; ${ }^{3}$ Department of Pharmacy, Affiliated Hospital of \\ Shaoxing University, Shaoxing 312000, China; ${ }^{4}$ Department of Pharmacy, Wuhan University, Renmin Hospital, Wuhan 430060, China \\ Contributions: (I) Conception and design: ZC Gu; (II) Administrative support: Y Qian; (III) Provision of study materials or patients: N Wang, NN \\ Shen; (IV) Collection and assembly of data: N Wang, NN Shen; (V) Data analysis and interpretation: ZC Gu, NN Shen; (VI) Manuscript writing: \\ All authors; (VII) Final approval of manuscript: All authors. \\ Correspondence to: Zhi-Chun Gu, MD. Department of Pharmacy, Renji Hospital, School of Medicine, Shanghai Jiao Tong University, Shanghai \\ 200127, China. Email: guzhichun213@163.com; Yan Qian, MD. Department of Pharmacy, The Second Affiliated Hospital of Chongqing Medical \\ University, Chongqing 400010, China. Email: cqqianyan@hospital.cqmu.edu.cn.
}

Background: Current evidence regarding the effectiveness and safety of direct oral anticoagulants (DOACs) in the elderly with atrial fibrillation (AF) remains scarce. Based on the emerging evidence from real-world studies (RWSs) associated with DOACs, we will perform a systematic review and meta-analysis of data from RWSs and randomized controlled trials (RCTs) to compare the effectiveness, safety and cost of DOACs versus Vitamin K antagonists (VKAs) in elderly patients with AF.

Methods: The MEDLINE, EMBASE and Cochrane Library databases will be systematically searched until June 30, 2019 for eligible RWSs and RCTs that reported the clinical outcomes between DOACs and VKAs in elderly patients with AF. The effectiveness outcome is stroke or systemic embolism (SE), and the safety outcomes are major bleeding, intracranial haemorrhage (ICH), gastrointestinal bleeding (GIB), myocardial infarction (MI) and all-cause mortality. A random-effects model will be used to calculate adjusted hazard ratios (HRs) for RWSs and relative risks (RRs) for RCTs, separately. The interaction analysis and the ratio of HRs (RHRs) will be applied to compare the treatment effect difference between RWSs and RCTs. A Markov model will be constructed to evaluate the cost-effectiveness of DOACs versus VKAs in elderly AF patients in real-world setting.

Discussion: This study will summarize all available evidences from RWSs and RCTs for a comprehensive and rigorous systematic review on the effectiveness and safety associated with DOACs, as well as perform a cost-effectiveness analysis to evaluate the price performance of DOACs among elderly AF patients in real clinical setting.

Trial registration: PROSPERO register platform (CRD42019142881, www.crd.york.ac.uk/PROSPERO/ display_record.php?RecordID =142881).

Keywords: Stroke; embolism; bleeding, real-world study; warfarin; dabigatran; rivaroxaban

Submitted Dec 13, 2019. Accepted for publication Feb 11, 2020.

doi: 10.21037/atm.2020.02.109

View this article at: http://dx.doi.org/10.21037/atm.2020.02.109 


\section{Introduction}

Advanced age is considered a significant risk factor for atrial fibrillation (AF) which is highly prevalent in the elderly, and AF prevalence estimate is $14 \%$ for individuals aged $>75$ years $(1,2)$. AF increases the risk for stroke by 5 -fold, and age $>75$ contributes to $25 \%$ of stroke occurrence $(3,4)$. Meanwhile, AF patients aged $\geq 75$ years is considered a risk factor for both $\mathrm{CHA}_{2} \mathrm{DS}_{2}$-VASc score and HAS-BLED score, thus the elderly is a risk factor for both stroke and bleeding. Anticoagulant therapy is an urgent intervention for reducing risk for stroke among elderly patients with AF. The oral anticoagulant Vitamin $\mathrm{K}$ antagonists (VKAs), due to the narrow therapeutic range, require therapeutic monitoring to ensure the ideal curative effects and avoid the risk for stroke and bleeding, thus leading to an incremental cost. Conversely, direct oral anticoagulants (DOACs: dabigatran, rivaroxaban, apixaban, and edoxaban) overcome the limitations of VKAs, with fewer drug and dietary interactions, and rapid onset of action. Based on these favorable practical advantages over VKAs, DOACs have been developed as the firstchoice for the thromboprophylaxis in AF (5-7). However, the potential benefits and harms profiles of DOACs have not been well established in elderly patients with AF, since elderly population are underrepresented, and the pivotal randomized controlled trials (RCTs) excluded these highrisk patients. Currently, the available results in elderly patients with $\mathrm{AF}$ are mainly from subgroup analysis (8-11), and no especially ad-hoc designed RCTs for elderly population have been performed to evaluate effectiveness and safety for DOACs. Although several prior meta-analysis have assessed the effects of DOACs in the elderly aged $>75$ years (12-15), the effectiveness and safety of DOACs remain uncertain due to the lack of adequate studies for individual agents and complete data from outcomes including intracranial haemorrhage (ICH), gastrointestinal bleeding (GIB), myocardial infarction (MI) and all-cause mortality. In addition, the pooled data from RCTs and realworld studies (RWSs) lead to dilute the power of RCTs (16), and a meta-analysis from all RWSs yielded borderline results (17), possibly leading to careful interpretation of these results. Several recent RWSs [Lip 2018 (18), Zoppellaro 2018 (19), Lai 2018 (20), Hohmann 2019 (21), Patti 2019 (22), Giustozzi 2019 (23), Chan 2019 (24), et al.] have provided new data and fueled systematical reassessment of the effectiveness and safety with DOACs. Meanwhile, because the cost of oral anticoagulants is an important consideration for long-term stroke prevention in elderly AF patients, it remains uncertain whether the higher DOACs costs will offset by potentially improved effectiveness and safety in real-world practice. We therefore will conduct a rigorous systematic review to compare the effectiveness and safety of DOACs versus VKAs between high-quality RWSs and RCTs, as well as will perform a cost-effectiveness analysis to assess the price performance of DOACs among elderly AF patients in real clinical setting.

\section{Methods}

\section{Study registration}

This study will be established in accordance with the PRISMA Statement and Cochrane Collaboration $(25,26)$. The corresponding protocol of this study has been registered in PROSPERO platform (CRD42019142881, www.crd.york.ac.uk/PROSPERO/display_record. php? RecordID =142881).

\section{Literature search}

A systematic search of MEDLINE, EMBASE, and Cochrane Library databases will be performed from inception to June 30, 2019 without language restriction. The search terms using medical subject heading (MeSH) and free text terms will be applied to conduct the search strategy. For the theme "atrial fibrillation", the terms used are: "atrial fibrillation" OR "AF". For the theme "DOACs", the terms used are: "dabigatran" OR "rivaroxaban" OR “apixaban” OR "edoxaban” OR "Non-vitamin K antagonist oral anticoagulants" OR "NOACs" OR "direct oral anticoagulants" OR "DOACs" OR "novel oral anticoagulants" OR "new oral anticoagulants" OR "factor Xa inhibitors" OR "factor IIa inhibitors". For the theme "VKAs", the terms used are: "Vitamin K antagonists" OR "VKAs" OR "warfarin" OR "coumadin" OR "coumadine" OR "dicoumarol" OR "dicoumarin" OR "acenocoumarol" OR "phenprocoumon”. The Boolean operator 'AND' will be used to combine the three comprehensive search themes. The detailed search strategy was available in Table 1 . The additional articles from reference lists will be also reviewed.

\section{Study selection}

Studies will be eligible for inclusion if they are RCTs or RWSs; included elderly patients (aged $\geq 75$ years) with AF; 
Table 1 Search strategy

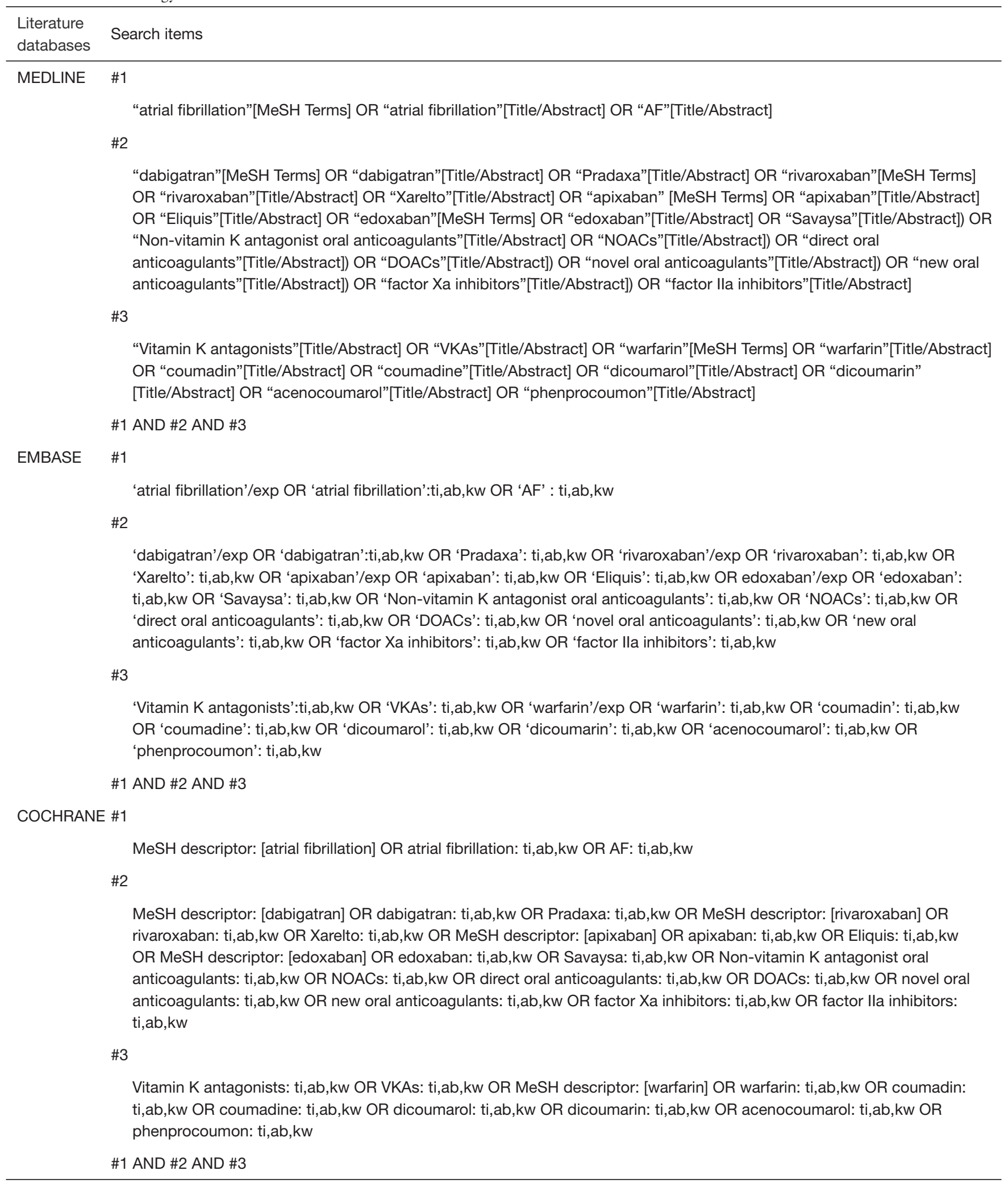


compared DOACs with VKAs; and reported benefit and harm outcomes [stroke or systemic embolism (SE), major bleeding, ICH, GIB, MI and all-cause mortality] from either main analyses or subgroup analyses. Studies concomitant with special clinical scenarios (dialysis, cancer, and diabetes mellitus) will be excluded. For the highest quality of RWSs, only studies that reported adjusted data using authorized method to minimize confounding [covariate adjustment (CA), propensity score adjustment (PSA), propensity score matching (PSM), inverse probability of treatment weighting (IPTW)] will be included. For outcome analyses in which studies have applied the same sources of data, we will only include studies which include the longest periods, unless the study periods do not overlap, or unless the studies include data from another source. We will exclude certain publications such as conference abstract and letter and studies that reported only crude data. Two researchers (Na Wang and Nan-Nan Shen) will independently screen all retrieved studies according to the inclusion criteria for further assessment, and any disagreements will be resolved by consultation or discussion with a third researcher (ZhiChun $\mathrm{Gu})$.

\section{Study outcomes}

The effectiveness outcome is stroke or SE, and the safety outcomes are major bleeding, ICH, GIB, MI and allcause mortality. Major bleeding was defined as a decrease in hemoglobin level of $2 \mathrm{~g} / \mathrm{dL}$ or greater within a 24 -hour period, or leading to a transfusion of 2 or more units of packed red cells, or requiring an additional endoscopy intervention, based on the International Society on Thrombosis and Hemostasis (ISTH) criteria (27). The cost outcomes include the number of quality-adjusted lifeyear (QALYs) and incremental cost-effectiveness ratio (ICER).

\section{Data extraction}

A priori designed form will be used to extract the data from included RWSs and RCTs. For RWSs, data extraction will include: (I) study characteristics (study name, data source and inclusion period, DOACs name and patient numbers, VKAs name and patient numbers, and adjusted method); (II) patient demographics and clinical characteristics [total number, mean age, percentage of female, percentage of heart failure, percentage of diabetes, percentage of stroke/ transient ischemic attacks, percentage of MI, percentage of renal disease, percentage of liver disease, percentage of anemia, percentage of cancer, $\mathrm{CHADS}_{2}$ score, $\mathrm{CHADS}_{2-}$ VASc score, HAS-BLED score, time in therapeutic range (TTR) for VKAs]; (III) patient bleeding history and concomitant drugs (angiotensin-converting enzyme inhibitors/angiotensin receptor blocker, calcium channel blocker, antiarrhythmic drug, estrogen, glucocorticoids, $\mathrm{H}_{2}-$ receptor antagonist, non-steroidal anti-inflammatory drug, proton pump inhibitor, serotonin receptor antagonist); (IV) effectiveness and safety outcome data [adjusted hazard ratios (HRs) and 95\% confidence intervals (95\% CIs) using CA, PSA, PSM, or IPTW method]. For RCTs, data extraction will include: (I) study characteristics (indication, NCT number, DOACs name, dose, patient numbers, VKAs name and patient numbers, and follow-up duration); (II) patient demographics and clinical characteristics (total number, mean age, percentage of female, weight, body mass index, percentage of heart failure, percentage of hypertension, percentage of diabetes, percentage of stroke/transient ischemic attacks, percentage of MI, percentage of cancer, creatinine clearance rate, $\mathrm{CHADS}_{2}$ score, antiplatelet drugs, TTR for VKAs); (III) effectiveness and safety outcome data (occurrence number and total number in DOACs and VKAs group). Owing to the probable overestimation of bleeding risk of DOACs versus VKAs, data from DOACsnaïve patients and VKAs-switchers will be extract separately, if available.

\section{Quality evaluation}

For RWSs, due to a higher risk of bias than RCTs, several factors in design and methods will be applied to mitigate bias when comparing outcomes between DOACs and VKAs: (I) used adjustment method for dealing with selection bias; (II) potential of residual confounding; (III) used methods for handling time-varying covariates and information censoring; (IV) reported baseline characteristics and outcome measures (28). Low, moderate, or high risk of bias will be applied to above each item. For RCTs, the methodological quality will be assessed for potential bias (low, unclear, high) according to the Cochrane Collaboration Risk of Bias Tool, which include selection bias (method of randomization and allocation concealment), information bias (masking of outcome adjudicators), and bias in the analysis (intention to treat analysis and completeness of follow-up) (29). Finally, quality assessment will be summarized as high, moderate, or low quality for each included RWS and RCT. 


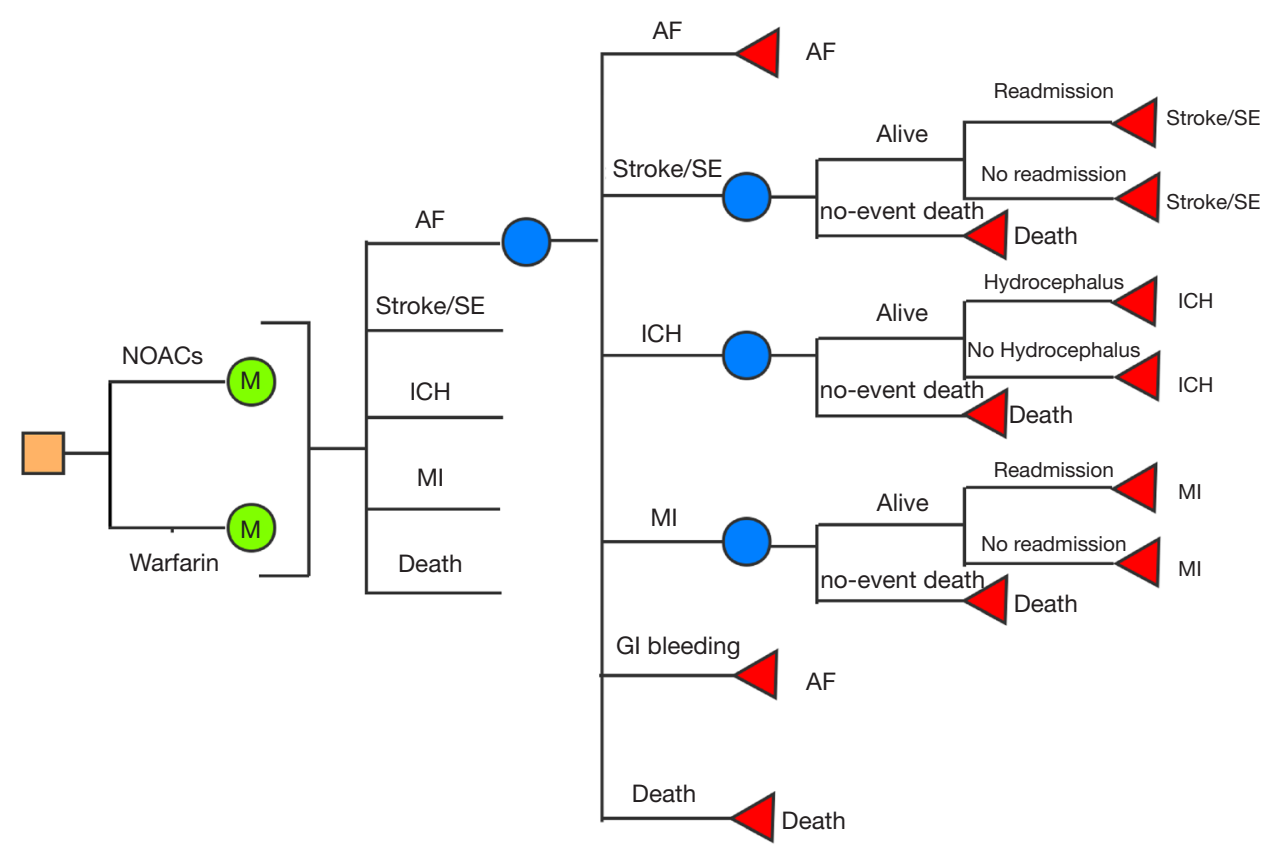

Figure 1 Schematic representation of the Markov model. AF, atrial fibrillation; SE, systemic embolism; ICH, intracranial haemorrhage; MI, myocardial infarction; GI bleeding, gastrointestinal bleeding.

\section{Statistical analysis}

The pooled analysis for RWSs and RCTs will be performed separately, and the forest plots will be used to show the results for included RWSs and RCTs with random-effects model. Meanwhile, both interaction analysis and the relative HRs (RHRs) will be applied to evaluate the comparability between RWSs and RCTs (30). To detect the reason of incomparability, further analysis between RWSs and RCTs will be conducted according to study characteristics, patient demographics, clinical characteristics, and clinical outcome definition of included studies. $\mathrm{I}^{2}$ statistic will be used to estimate the heterogeneity, where $\mathrm{I}^{2}>50 \%$ indicating high heterogeneity (31). The source of high heterogeneity will be discussed based on subsequent subgroup analyses and sensitivity analyses. For RWSs, The adjusted hazard ratios (HRs) with their 95\% CI will be pooled, and the subgroup analyses will be performed on the basis of individual agents (rivaroxaban, apixaban, edoxaban, and dabigatran), different dosage (standard dose and low dose), varying TTR for VKAs (TTR $>65 \%$ or $<65 \%$ ), gender (women and men), very elderly population (the hierarchy of $>80,>85$, and $>90$ years respectively), DOACs-naïve patients or VKAsswitchers, and countries or regions (U.S.A, Canada, Italy, Germany, Sweden, Danish, et al.). For RCTs, relative risks (RRs) and associated 95\% CI will be calculated. And subgroup analyses will be conducted according to individual agents (rivaroxaban, apixaban, edoxaban, and dabigatran), dose (standard dose and low dose), and follow-up duration (>12 or $<12$ months). In order to detect the robustness of the results, the sensitivity analyses will be conducted by sequential elimination of each study and excluding low quality studies to determine whether these excluded studies affect the conclusions. Publication bias will be evaluated by using funnel plots and further quantification by using Begg's test as well as Egger's test (26). Meta-regression analysis will also be performed to determine the potential bias of effect factors on outcomes. All statistics will be performed by using STATA software (version13, Statacorp, College Station, Texas, USA).

\section{Cost-effectiveness analysis}

\section{Decision analytic model}

A decision state-transition Markov model with one-year cycles and 10-year horizon will be created to compare DOACs with VKAs among AF population aged over 75. A simplified schematic representation of the model structure is shown in Figure 1. Six mutually exclusive health states 
are included in this model: $\mathrm{AF}$ without complications, $\mathrm{AF}$ with stroke/SE, AF with ICH, AF with GIB, AF with MI, and death. The patients first starting a DOAC or VKA are assumed to start in the state of $\mathrm{AF}$, and time continue to elapse until the patients end up in the death state or the executions of cycle counted to 10 . It is assumed that during each cycle, patient may remain in their current health state or experience an event that will cause them to move to at most one subsequent state. Patients experiencing the first non-fatal stroke, MI, or ICH are assumed to experience only one 30-day readmission in each cycle. Cost analyses will be performed by using TreeAge Pro 2011 software (TreeAge Software, Inc., MA, USA).

\section{Sources of treatment estimates}

In current cost-effectiveness analysis, data from DOACs or VKAs- naïve patients will be used. The baseline rates of various clinical events in patients taking VKAs will be obtained from the results of included RWSs. HRs will be applied to represent the comparative risks of such events for the DOACs relative to VKAs, which will be calculated from the present meta-analyses of RWSs. The rates of 30-day readmission for clinical events will be obtained from the published literatures (32-34). These readmission rates will be adjusted to better reflect the old population by applying HRs. The age-dependent all-cause mortality figures will be drawn from the Global Burden of Disease Study in 2017 (GBD 2017). These will be assumed to be the same in VKAs and DOACs. All clinical event rates will be finally converted into probabilities per cycle and then be inputted to the model.

\section{Health state utilities and cost}

The main outcome of this analysis is QALYs gained which incorporate both the quality and quantity of life lived. Health utility values that describe the quality of life for different health states will be obtained from the published researches and range from 0 (death) to 1 (perfect health). The adjusted weight of 0.82 will be applied to calculate the baseline utilities of subjects aged $>75$ years (35). Onetime dis-utilities will be applied for acute events $(-0.138$ for stroke, -0.181 for ICH, -0.182 for GIB, -0.125 for MI) in each cycle. Anticoagulant therapies are also assumed to cause the slightly derogation of the health utilities, which are assessed to be -0.002 and -0.03 for DOACs and VKAs. The baseline utilities will be assumed to decrease $2 \%$ per year to explain the decline of health with advancing age (36).

The model will incorporate all direct healthcare costs for the therapies and treatment of associated acute clinical events, as well as costs for long-term care after experiencing the first non-fatal events. The costs for clinical events will be obtained from the published literatures and inflated to 2019 dollars. All the input costs will be discounted at 3.5\% annually for eliminating the inflation effects.

Cost-effectiveness will be assessed by using the commonly accepted willingness in US for paying threshold of \$50,000 per QALY gained, and be reported as ICER (37). The ICER provides a standardized approach to measure cost per unit of health improvement in and across health states. Cost-effectiveness is assessed annually to determine at which point in time treatment options achieved accepted levels of cost-effectiveness.

\section{Sensitivity analysis}

Sensitivity analysis will be conducted to evaluate the impact of uncertain parameters on primacy result. All model parameters will be varied over their $95 \%$ CIs. For the data without $95 \%$ CI provided, a variation at $\pm 5 \%, \pm 10 \%$ and $\pm 20 \%$ will be assumed for the parameters of utility, probability and cost. All the clinical outcomes and health utilities will be assigned as beta distributions. Gamma or Log-normal distributions will be assumed for drugs and healthcare costs.

\section{Discussion}

Several previous systematic reviews and meta-analyses have assessed the efficacy and safety of DOACs in elderly patients with AF. However, there are limitations in these studies. The earliest meta-analysis only pooled available data from RCTs, which demonstrated a favorable safety of DOACs in stroke prevention (12). However, edoxaban, one of the DOACs, was not included in this study, and the classification of major or clinically relevant bleeding may lead to the inaccurate estimation for bleeding risk. In 2015, an updated meta-analysis indicated the superior efficacy in reducing thrombotic risk for DOACs (13), but bleeding outcomes were various. It is worth noting that dabigatran increased the risk for GIB when compared to VKAs. Although this study obtained all available evidence of RCTs including edoxaban, there were two significant limitations: several summary results in individual DOAC were based on only 1 or 2 studies, therefore inevitably leading to publication bias; and the outcome data on cardiovascular events, in particular, MI and all-cause mortality, were not 
reported. Up to now, only one systematic review pooled evidence for both RCTs and RWSs in older patients (16). This study indicated that DOACs were superior to VKAs in stroke prevention, with reduced risk for major bleeding. However, the risk for major bleeding was inconsistent between RCTs and RWSs. Moreover, the evidence of patients aged $65-74$ years was also involved in this study, which may underestimate the risk for bleeding. The similar results were presented in subsequent meta-analysis $(14,15,38)$, DOACs use demonstrated a reduced risk for stroke and the undifferentiated risk for major bleeding in elderly patients when compared to VKAs. Nevertheless, the main limitations of these several studies included: the limited number of studies with individual DOAC was analyzed, leading to the lack of insufficient estimation for individual DOAC; the pooled results were mainly from subgroup data, thus the interpretation required caution; furthermore, in some studies, since the lack of baseline patients characteristics, the anticoagulant effects on patients with other comorbidities or risk factors remained unknown. Based on the above limitations, there is the need for obtaining evidence from patients in the realworld settings and reassess this topic. With the gradual emergence of real-world data for DOACs, a recent metaanalysis of 20 observational studies (17), reported the reduced risk for ischemic stroke and ICH in the elderly patients with DOACs versus VKAs, and no significant difference on major bleeding, MI and all-cause mortality. Although this was the comprehensive analysis of available data from RWSs, which clarified the effectiveness and safety of DOACs versus VKAs in AF patients aged $>75$ years, the majority of pooled risk estimate for effectiveness (composite outcomes of all strokes, or transient ischemic attack, or other thromboembolic events) and safety (composite outcomes of major bleeding, GIB, and ICH) were borderline (fixed-effects models: HR: 0.95, 95\% CI: 0.91-0.99 for effectiveness; HR: 0.97, 95\% CI: 0.93-1.00 for safety; HR: 0.95; random-effects models: HR: 0.93, 95\% CI: 0.85-1.01 for effectiveness; HR: 0.95, 95\% CI: $0.87-$ 1.04 for safety). Among the included studies in this metaanalysis, only one study examined the risk for mortality of reduced dose DOACs compared with VKAs among elderly patients with AF (39), therefore may lead to reduced risk outcome. In addition, another study (Forslund 2017) reported all-dose, low-dose, and standard-dose DOACs (40), Mitchell and colleagues repeatedly pooled data, and the evidence of edoxaban was not involved in this metaanalysis. Regarding the above limitations, the robustness and reliability of the results are affected. Certainly, the price performance of DOACs versus VKAs remains uncertain. Therefore, the generalizable and sufficient data sets are essential to provide a systematical estimation in elderly patients with AF. We will perform a systematic review to provide the most comprehensive evaluation of effectiveness and safety and cost analysis of DOACs in AF patients aged $>75$ years, by simultaneously including currently available data from RWSs and RCTs. The evidence derived from RWSs and their meta-analyses will facilitate validation of conclusions drawn from RCTs and reassure decision-makers that findings can be extrapolated to real-world populations.

\section{Acknowledgments}

Funding: This work was supported by Internet-based development and application of stroke management and service platform in atrial fibrillation patients (No. cstc2017shmsA130041), Shanghai municipal heath commission (20184Y0022), Cultivation fund of clinical study of Renji hospital (PY2018-III-06), General scientific project of Zhejiang education department (Y201941020), the Program of Shaoxing science and technology bureau (2017B70010), and Clinical Pharmacy Innovation Research Institute of Shanghai Jiao Tong University School of Medicine (CXYJY2019ZD001, CXYJY2019QN004).

\section{Footnote}

Conflicts of Interest: The authors have no conflicts of interest to declare.

Ethical Statement: The authors are accountable for all aspects of the work in ensuring that questions related to the accuracy or integrity of any part of the work are appropriately investigated and resolved. Ethical approval is not necessary because this study is a protocol of systematic review and meta-analysis.

Open Access Statement: This is an Open Access article distributed in accordance with the Creative Commons Attribution-NonCommercial-NoDerivs 4.0 International License (CC BY-NC-ND 4.0), which permits the noncommercial replication and distribution of the article with the strict proviso that no changes or edits are made and the original work is properly cited (including links to both the formal publication through the relevant DOI and the license). See: https://creativecommons.org/licenses/by-nc-nd/4.0/. 


\section{References}

1. Lloyd-Jones DM, Wang TJ, Leip EP, et al. Lifetime risk for development of atrial fibrillation: the Framingham Heart Study. Circulation 2004;110:1042-6.

2. Lip GYH, Brechin CM, Lane DA. The global burden of atrial fibrillation and stroke: a systematic review of the epidemiology of atrial fibrillation in regions outside North America and Europe. Chest 2012;142:1489-98.

3. Lip GY, Nieuwlaat R, Pisters R, et al. Refining clinical risk stratification for predicting stroke and thromboembolism in atrial fibrillation using a novel risk factor-based approach: the euro heart survey on atrial fibrillation. Chest 2010;137:263-72.

4. Zoni-Berisso M, Lercari F, Carazza T, et al. Epidemiology of atrial fibrillation: European perspective. Clin Epidemiol 2014;6:213-20.

5. Freedman B, Potpara TS, Lip GY. Stroke prevention in atrial fibrillation. Lancet 2016;388:806-17.

6. Marzec LN, Wang J, Shah ND, et al. Influence of Direct Oral Anticoagulants on Rates of Oral Anticoagulation for Atrial Fibrillation. J Am Coll Cardiol 2017;69:2475-84.

7. Steffel J, Verhamme P, Potpara TS, et al. The 2018 European Heart Rhythm Association Practical Guide on the use of non-vitamin $\mathrm{K}$ antagonist oral anticoagulants in patients with atrial fibrillation. Eur Heart J 2018;39:1330-93.

8. Eikelboom JW, Wallentin L, Connolly SJ, et al. Risk of bleeding with 2 doses of dabigatran compared with warfarin in older and younger patients with atrial fibrillation: an analysis of the randomized evaluation of long-term anticoagulant therapy (RE-LY) trial. Circulation 2011;123:2363-72.

9. Halperin JL, Hankey GJ, Wojdyla DM, et al. Efficacy and safety of rivaroxaban compared with warfarin among elderly patients with nonvalvular atrial fibrillation in the Rivaroxaban Once Daily, Oral, Direct Factor Xa Inhibition Compared With Vitamin K Antagonism for Prevention of Stroke and Embolism Trial in Atrial Fibrillation (ROCKET AF). Circulation 2014;130:138-46.

10. Halvorsen S, Atar D, Yang H, et al. Efficacy and safety of apixaban compared with warfarin according to age for stroke prevention in atrial fibrillation: observations from the ARISTOTLE trial. Eur Heart J 2014;35:1864-72.

11. Kato ET, Giugliano RP, Ruff CT, et al. Efficacy and Safety of Edoxaban in Elderly Patients With Atrial Fibrillation in the ENGAGE AF-TIMI 48 Trial. J Am Heart Assoc 2016. doi: 10.1161/JAHA.116.003432.
12. Sardar P, Chatterjee S, Chaudhari S, et al. New oral anticoagulants in elderly adults: evidence from a meta-analysis of randomized trials. J Am Geriatr Soc 2014;62:857-64.

13. Sharma M, Cornelius VR, Patel JP, et al. Efficacy and Harms of Direct Oral Anticoagulants in the Elderly for Stroke Prevention in Atrial Fibrillation and Secondary Prevention of Venous Thromboembolism: Systematic Review and Meta-Analysis. Circulation 2015;132:194-204.

14. Caldeira D, Nunes-Ferreira A, Rodrigues R, et al. Nonvitamin $\mathrm{K}$ antagonist oral anticoagulants in elderly patients with atrial fibrillation: A systematic review with metaanalysis and trial sequential analysis. Arch Gerontol Geriatr 2019;81:209-14.

15. Kim IS, Kim HJ, Kim TH, et al. Non-vitamin K antagonist oral anticoagulants have better efficacy and equivalent safety compared to warfarin in elderly patients with atrial fibrillation: A systematic review and metaanalysis. J Cardiol 2018;72:105-12.

16. Bai Y, Guo SD, Deng H, et al. Effectiveness and safety of oral anticoagulants in older patients with atrial fibrillation: a systematic review and meta-regression analysis. Age Ageing 2018;47:9-17.

17. Mitchell A, Watson MC, Welsh T, et al. Effectiveness and Safety of Direct Oral Anticoagulants versus Vitamin K Antagonists for People Aged 75 Years and over with Atrial Fibrillation: A Systematic Review and Meta-Analyses of Observational Studies. J Clin Med 2019. doi: 10.3390/ jcm8040554.

18. Lip GYH, Keshishian A, Li X, et al. Effectiveness and Safety of Oral Anticoagulants Among Nonvalvular Atrial Fibrillation Patients. Stroke 2018;49:2933-44.

19. Zoppellaro G, Zanella L, Denas G, et al. Different safety profiles of oral anticoagulants in very elderly non-valvular atrial fibrillation patients. A retrospective propensity score matched cohort study. Int J Cardiol 2018;265:103-7.

20. Lai CL, Chen HM, Liao MT, et al. Dabigatran, Rivaroxaban, and Warfarin in the Oldest Adults with Atrial Fibrillation in Taiwan. J Am Geriatr Soc 2018;66:1567-74.

21. Hohmann C, Hohnloser SH, Jacob J, et al. Non-Vitamin K Oral Anticoagulants in Comparison to Phenprocoumon in Geriatric and Non-Geriatric Patients with Non-Valvular Atrial Fibrillation. Thromb Haemost 2019;119:971-80.

22. Patti G, Pecen L, Lucerna M, et al. Net Clinical Benefit of Non-Vitamin K Antagonist vs Vitamin K Antagonist Anticoagulants in Elderly Patients with Atrial Fibrillation. Am J Med 2019;132:749-57.e5.

23. Giustozzi M, Vedovati MC, Verso M, et al. Patients aged 
90years or older with atrial fibrillation treated with oral anticoagulants: A multicentre observational study. Int J Cardiol 2019;281:56-61.

24. Chan YH, Lee HF, See LC, et al. Effectiveness and Safety of Four Direct Oral Anticoagulants in Asian Patients With Nonvalvular Atrial Fibrillation. Chest 2019;156:529-43.

25. Wei AH, Gu ZC, Zhang C, et al. Increased risk of myocardial infarction with dabigatran etexilate: fact or fiction? A critical meta-analysis of over 580,000 patients from integrating randomized controlled trials and realworld studies. Int J Cardiol 2018;267:1-7.

26. Liberati A, Altman DG, Tetzlaff J, et al. The PRISMA statement for reporting systematic reviews and metaanalyses of studies that evaluate health care interventions: explanation and elaboration. J Clin Epidemiol 2009;62:e1-34.

27. Schulman S, Angeras U, Bergqvist D, et al. Definition of major bleeding in clinical investigations of antihemostatic medicinal products in surgical patients. J Thromb Haemost 2010;8:202-4.

28. Gu ZC, Wei AH, Zhang C, et al. Risk of Major Gastrointestinal Bleeding With New vs Conventional Oral Anticoagulants: A Systematic Review and Meta-analysis. Clin Gastroenterol Hepatol 2019. [Epub ahead of print].

29. Higgins JP, Altman DG, Gotzsche PC, et al. The Cochrane Collaboration's tool for assessing risk of bias in randomised trials. BMJ 2011;343:d5928.

30. Coscia C, Jaureguizar A, Quezada CA, et al. Comparison of All-Cause Mortality Following VTE Treatment Between Propensity Score-Adjusted Observational Studies and Matched Randomized Controlled Trials: MetaEpidemiologic Study. Chest 2019;155:689-98.

31. Higgins JP, Thompson SG, Deeks JJ, et al. Measuring inconsistency in meta-analyses. BMJ 2003;327:557-60.

32. Adil SM, Liu B, Charalambous LT, et al. Healthcare Economics of Hydrocephalus After Aneurysmal

Cite this article as: Wang N, Shen NN, Wu Y, Zhang C, Pan MM, Qian Y, Gu ZC. Comparison of effectiveness and safety of direct oral anticoagulants versus vitamin-k antagonists in elderly patients with atrial fibrillation: a systematic review and cost-effectiveness analysis protocol. Ann Transl Med 2020;8(6):391. doi: 10.21037/atm.2020.02.109
Subarachnoid Hemorrhage in the United States. Transl Stroke Res 2019;10:650-63.

33. Kim LK, Yeo I, Cheung JW, et al. Thirty-Day Readmission Rates, Timing, Causes, and Costs after STSegment-Elevation Myocardial Infarction in the United States: A National Readmission Database Analysis 20102014. J Am Heart Assoc 2018;7:e009863.

34. Vahidy FS, Donnelly JP, McCullough LD, et al. Nationwide Estimates of 30-Day Readmission in Patients With Ischemic Stroke. Stroke 2017;48:1386-8.

35. Reddy VY, Akehurst RL, Gavaghan MB, et al. CostEffectiveness of Left Atrial Appendage Closure for Stroke Reduction in Atrial Fibrillation: Analysis of Pooled, 5-Year, Long-Term Data. J Am Heart Assoc 2019;8:e011577.

36. Fryback DG, Dunham NC, Palta M, et al. US norms for six generic health-related quality-of-life indexes from the National Health Measurement study. Med Care 2007;45:1162-70

37. Grosse SD. Assessing cost-effectiveness in healthcare: history of the \$50,000 per QALY threshold. Expert Rev Pharmacoecon Outcomes Res 2008;8:165-78.

38. Malik AH, Yandrapalli S, Aronow WS, et al. MetaAnalysis of Direct-Acting Oral Anticoagulants Compared With Warfarin in Patients > 75 Years of Age. Am J Cardiol 2019;123:2051-7.

39. Nielsen PB, Skjoth F, Sogaard M, et al. Effectiveness and safety of reduced dose non-vitamin $\mathrm{K}$ antagonist oral anticoagulants and warfarin in patients with atrial fibrillation: propensity weighted nationwide cohort study. BMJ 2017;356:j510.

40. Forslund T, Wettermark B, Andersen M, et al. Stroke and bleeding with non-vitamin $\mathrm{K}$ antagonist oral anticoagulant or warfarin treatment in patients with non-valvular atrial fibrillation: a population-based cohort study. Europace 2018;20:420-8. 